\title{
The Ephi System for VLF Direction Finding*
}

\author{
G. Hefley, R. F. Linfield, and T. L. Davis
}

(August 11, 1960)

\begin{abstract}
A new system of VLF direction finding has been developed and tested by the National Bureau of Standards, Boulder, Colorado. The system has been named "Ephi" (E- $\phi)$ because the bearing of the transient signal is determined from the relative phase $(\phi)$ of the vertical electrical field $(\mathrm{E})$ received at spaced antennas. The advantage of this scheme compared to conventional crossed-loop techniques is that it minimizes siting and polarization errors. A minimum of three antennas must be used to resolve directional ambiguity, and the preferrred antenna location is at the vertices of an equilateral triangle with baselines equal to $1 / 3$ to $1 / 10$ wavelength (at $10 \mathrm{kc}$ ). Appropriate phase detectors, delay lines and coincidence circuits are used to obtain a directional code in preset sectors. Within practical instrumentation limits any number of sectors of variable widths can be operated simultaneously and each can be rotated in azimuth.
\end{abstract}

\section{Introduction}

Direction-finding techniques used for the location of transient signals (such as sferies) usually employ crossed-loop antennas. The accuracy of such systems is limited largely by the siting and polarization errors involved. ${ }^{1,2}$ A system of direction finding which has been developed by the National Bureau of Standards minimizes these errors, and has certain other features which make it particularly useful in various types of sferics studies. The new system has been named "Ephi" $(\mathrm{E}-\phi)$ because the bearing of the transient signal is determined from the relative phase $(\phi)$ of the vertical electric field (E) received at spaced antennas.

In this system, spaced vertical antennas replace the loops and a minimum of three antennas must be used to resolve directional ambiguity. The antennas are closely spaced, preferably in the range of $1 / 3$ to $1 / 10$ wavelength. The relative time of arrival or phase of a signal at the antennas depends on the geometric configuration of the antennas and the direction of the signal source. In general, the optimum configuration for three antennas is at the vertices of an equilateral triangle. In this case, the sides of the triangle define the baselines of three hyperbolic grids with a mutual azimuthal spacing of 120 degrees.

Since the antenna spacing is a small fraction of a wavelength, differences in the immediate environment of the antennas are minimized. Experimental results thus far have not revealed any siting or environmental errors even when the individual antenna sites were purposely chosen with radical dissimilarities. The present experimental evidence, however, is not adequate to establish positively that siting errors are eliminated in this system.

\footnotetext{
*Contribution from the Central Radio Propagation Laboratory, National Bureau of Standards, Boulder, Colo.

$1 \mathrm{~F}$. Horner, The accuracy of the location of sources of atmospheries by radio direction-finding, Proc. IEE, 101, 383, (1954).

2 H. G. Hopkins and B. G. Pressey, Current direction-finding practice, Proc. IEE, 105, 307, (1958).
}

A unique aspect of the Ephi system is that the relative time of arrival of signals from any direction or number of directions is anticipated rather than measured after recording. When finite tolerances are placed on the relative times of arrival which are anticipated, signals are accepted from a sector whose width is governed by the tolerances. Whenever a signal is received, a sector identification code is generated which may be used for selectively recording data with respect to direction. Within practical instrumentation limits any number of sectors of different widths can be operated simultaneously and independently of each other.

\section{System Geometry}

In a system using three antennas in the configuration of an equilateral triangle, the distance from a signal source to each antenna (fig. 1) is defined by the following equations:

$$
\begin{aligned}
& d_{A}=\sqrt{R^{2}+r^{2}-2 r R \cos \theta} \\
& d_{B}=\sqrt{R^{2}+r^{2}-2 r R \cos (120-\theta)} \\
& d_{C}=\sqrt{R^{2}+r^{2}-2 r R \cos (120+\theta)} .
\end{aligned}
$$

The differences in the time of arrival of the signal at the antennas are proportional to the differences in the distance to the source.

$$
\begin{aligned}
& \left(d_{B}-d_{A}\right) k=T_{B-A} \\
& \left(d_{C}-d_{B}\right) k=T_{C-B} \\
& \left(d_{C}-d_{A}\right) k=T_{C-A}
\end{aligned}
$$

where $k \approx 5.37 \mu \mathrm{sec} / \mathrm{stat}$ te mile, the inverse velocity of radio wave propagation near the surface of the earth. 


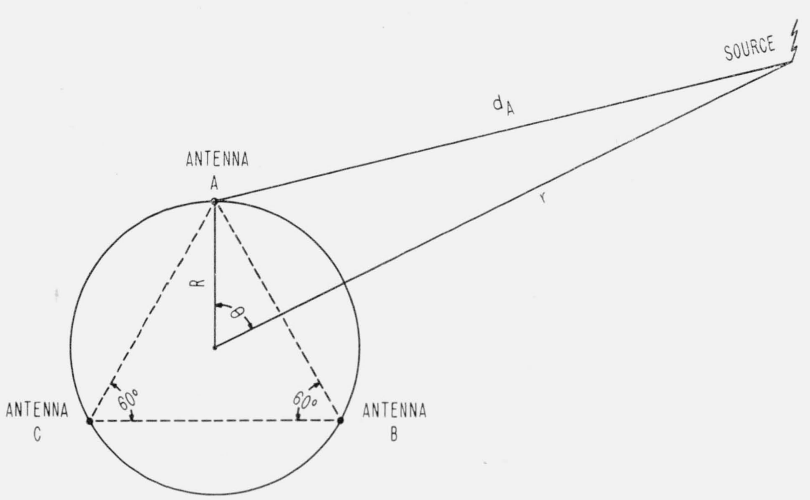

Figure 1. System geometry.

It is convenient to let the baseline $R \sqrt{3}$ equal unity. Figure 2 is a polar plot of the time or distance differences normalized to a baseline of unit length, with $r \gg R$. The exact values of these differences obviously depend on the ratio $r / R$, but when the distance to the source is 10 baseline units or more the condition $r \gg R$ is satisfied for most purposes. At a distance of 10 baseline units the values shown in the figure are in error by approximately 0.5 percent.

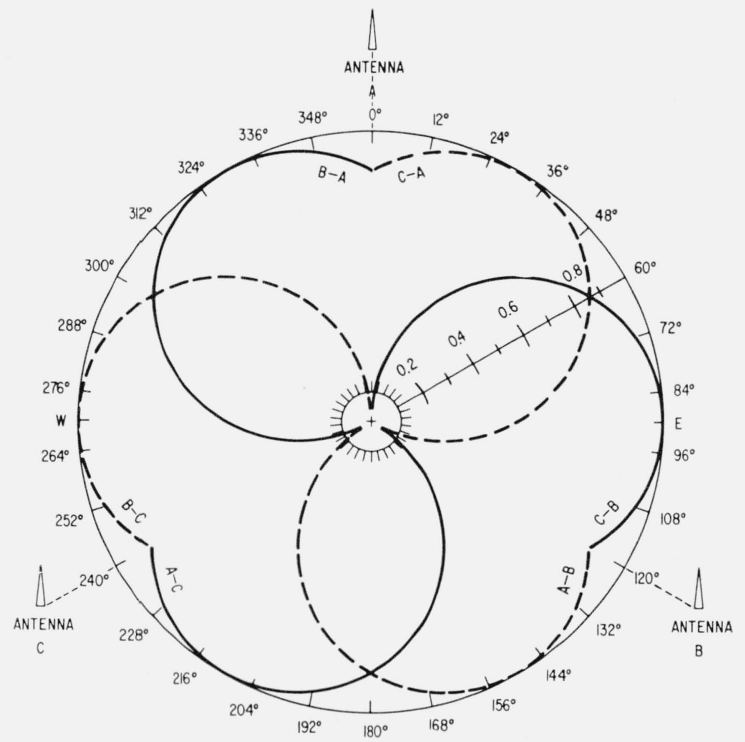

Figure 2. Normalized time or distance differences.

Since the antennas are closely spaced the differences in arrival time are small. It is, therefore, necessary to resolve these small differences rather precisely in order to provide satisfactory azimuthal sensitivity. The means by which this resolution is accomplished and the technique by which the direction of a signal is anticipated are interrelated and can best be explained together.

The significant steps in the process are:

1. Identical antennas and band-pass filters are used to derive pulses from the transient signals. A relatively large bandwidth (order of $60 \%$ ) is required to provide short pulse rise and decay times.
2. The phase of the sinusoidal waveform or pulse is used to define the arrival time of the signal. The first axis crossing of the waveform is used to define a precise point in time which is independent of the amplitude of the signal, and a very short trigger is generated at this point.

3. A signal from a particular azimuth is recognized by introducing the delays required to produce coincidence. The early triggers are delayed in time until they are coincident with the trigger from the most distant antenna. These triggers then initiate rectangular waveforms as shown in figure 3 . The time coincidence of the three waveforms is detected by circuitry which generates a new trigger. This indicates the occurrence of a signal at a particular azimuth and is produced as long as all three waveforms have full amplitude at any one time. The duration of the rectangular waveform, therefore, determines the width of the sector in which the signal must originate to produce a coincidence trigger.

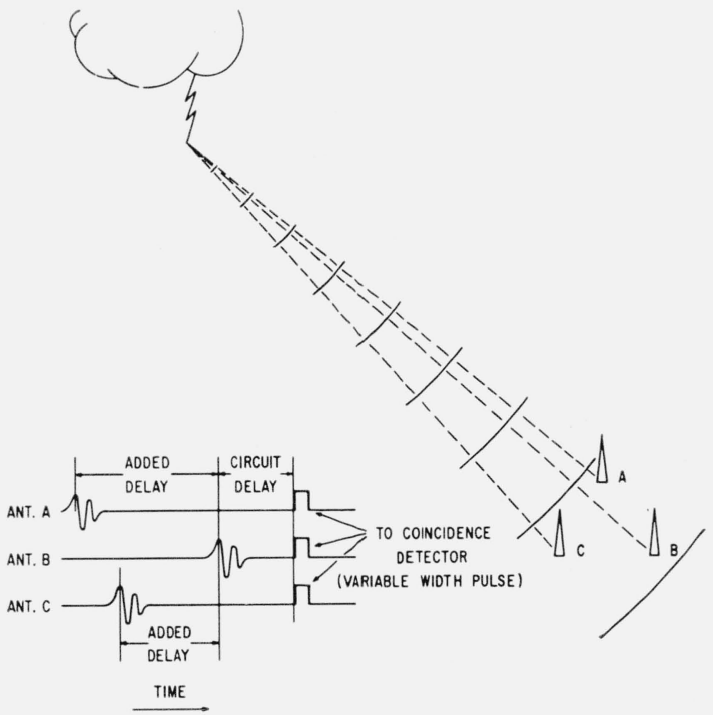

Figure 3. Sector determination.

The pulse width is also directly related to the baseline length. For example a 1-deg sector requires a pulse width of approximately $0.05 \mu \mathrm{sec}$ with a 1-mile baseline, but the same sector width can be obtained with a $0.25 \mu$ sec pulse if a 5 -mile baseline is used. The advantages of the longer baseline from an instrumentation standpoint are obvious.

The linear relationship between sector width and pulse width holds only for relatively small sector widths (less than about $12 \mathrm{deg}$ ), but since it is usually desirable to employ much narrower sectors the nonlinear effects will not be discussed.

Certain secondary azimuthal and range errors also limit bearing accuracy. There is a small cyclic change in sector width which varies with the azimuth setting, and another source of inaccuracy arises from the use of one set of delays for all ranges. In the experimental system the delays were computed for a 5,000 mile range. This introduces a calculable error 
at other ranges but it is significant only in the vicinity of the antenna system. The error in bearing may amount to several degrees for sources only a baseline length away, but when the distance to the source is greater than about 10 baseline lengths the error becomes so small it may be neglected. With further refinement these systematic azimuthal errors could be reduced or eliminated by introducing appropriate correcting circuits in the equipment.

\section{Instrumentation}

The circuitry which is necessary to accomplish the basic functions described in the preceding section involves a very high degree of phase matching stability. The functional scheme of the system is shown in figure 4. The experimental equipment installed near Brighton, Colo. (about 20 miles northeast of the Denver airport) is shown in figure 5.

In the choice of antennas, two considerations are of major importance. First, the mutual impedance among the antennas must be negligible and second, the complex impedance of each antenna must be made as insensitive as possible to environmental changes. The mutual impedance is minimized by physical separation of the antennas and by extremely loose coupling between the antennas and the resonant circuits (see fig. 6).

The antennas which have been used to date are of the type shown in figure 7 . The height is $125 \mathrm{ft}$ and the measured capacity is approximately $515 \mu \mu f$.

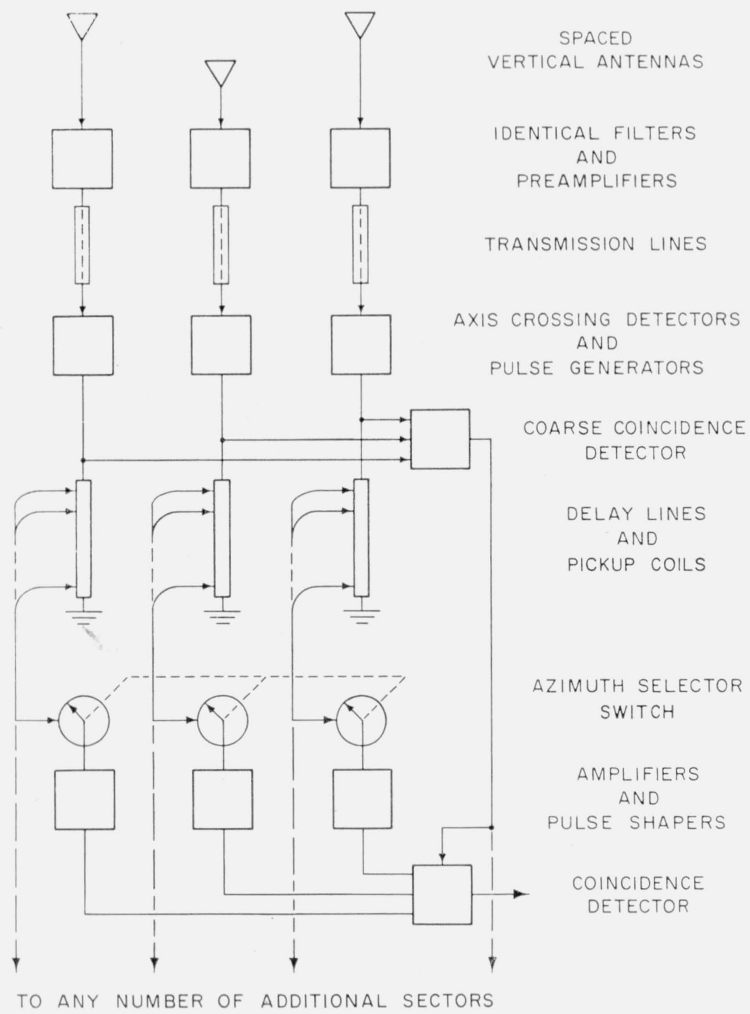

Figure 4. Functional system diagram.

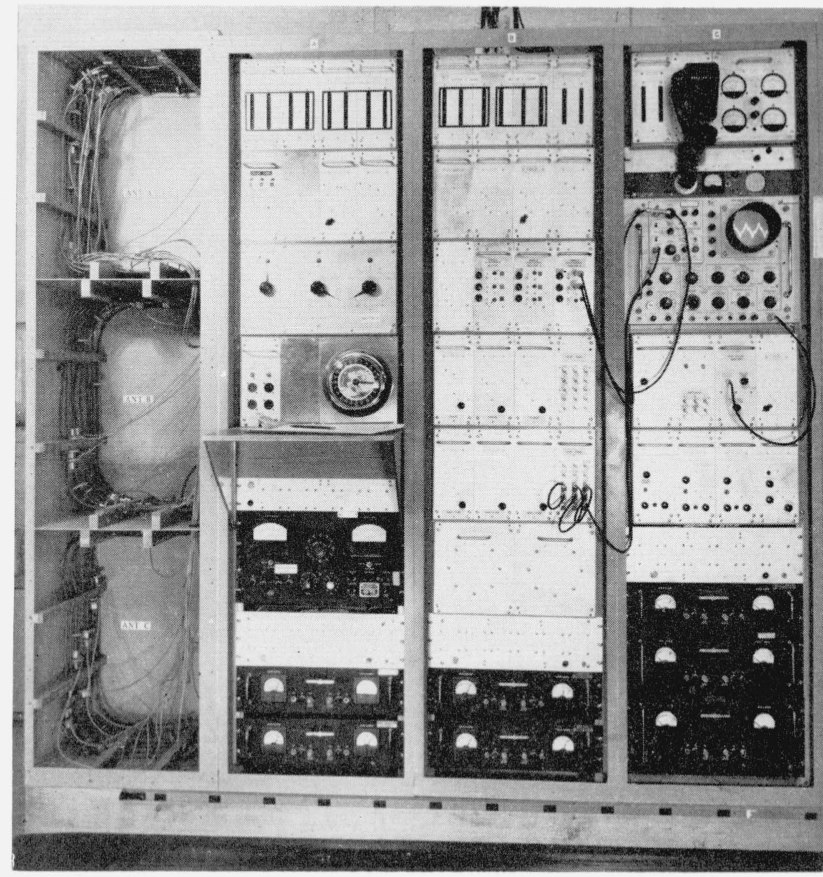

Figure 5. Ephi equipment at Brighton station.

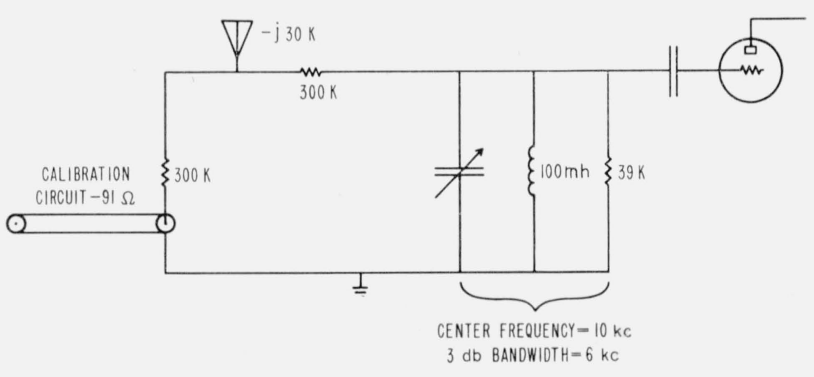

Figure 6. Antenna coupling circuit.

When an antenna of these proportions is used, the presence of a person near the base has very little effect on the antenna characteristics. Some further practical advantages of such a large antenna are that the magnitude of its impedance makes it more feasible to use passive circuit elements prior to the first grid thus reducing intermodulation by undesired signals. By virtue of the large effective height, very loose coupling can be used without degrading the overall noise figure of the receiving system.

The principal requirements for the preamplifiers, located at the base of each antenna, are large dynamic range and high power. The large dynamic range is necessary because of the widely varying amplitudes of the transient signals. High power is needed to insure a good signal-to-noise ratio at the receiving end of the transmission lines. While coaxial cable is used, it is not perfectly shielded and is, therefore, subject to pickup from power lines, and also the same transient signals received by the antennas induce small voltages in the transmission lines. 


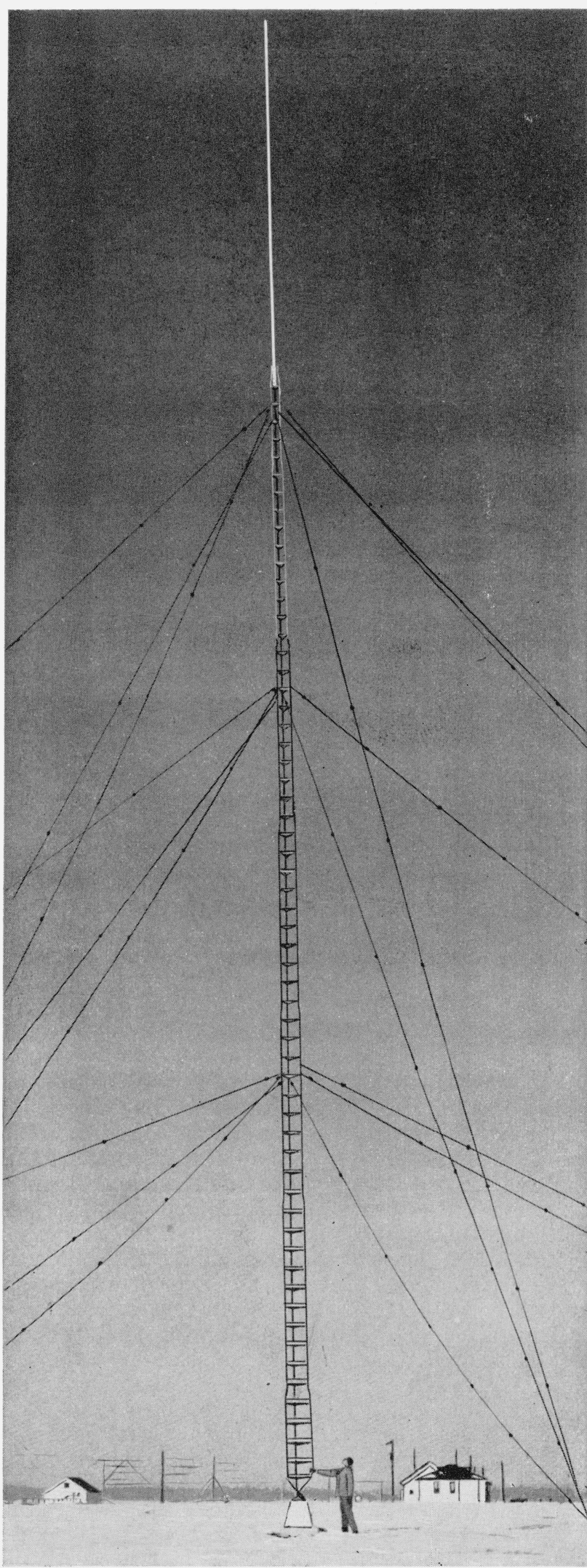

Figure 7. Ephi system antenna.
These voltages are similar to those from the antennas but are not in the same phase. As a result it is especially important that the undesired pickup be made negligible compared to the desired signals from the antennas.

The transmission lines leading from the preamplifiers to the centrally located equipment are adjusted so that transmission times are equal. These lines feed identical axis-crossing detectors and pulse generators. The basic functions of these circuits were described in the preceding section, but are shown in greater detail in figure 8 . The first axis crossing of detectable amplitude defines the arrival time of the signal. The band-pass filtering which is done in part at the preamplifier and completed at the input to the axis-crossing detector produces a sinusoidal waveform of the nature shown in figure 8 . Over a wide range of input levels the first axis crossing occurs within the linear range of the amplifiers. Saturation at the later axis crossings does not affect the amplifiers since these crossings are rejected by the coarse coincidence circuit.

The different steps in the coincidence detection process are shown in figure 9. The coarse coincidence detector selects the first complete set of axiscrossing triggers. In general, the first set will be complete, but regardless of how perfectly the three separate circuits are matched, a number of critical input levels exist which determine which axis crossing will produce the first trigger. At such critical levels all the axis-crossing detectors may not produce the first trigger at the same crossing as illustrated by the dotted trigger in figure $9 \mathrm{~b}$.

The duration of the coarse coincidence pulse is made somewhat greater than the maximum time difference. The coarse coincidence detection is, therefore, not a function of azimuth.

As indicated in figure $9 \mathrm{~d}$, when all four coincidence pulses are added, the combined amplitudes exceed a threshold. When this occurs a new trigger is generated which is used to signify the occurrence of a signal in that sector. To increase the reliability of the coincidence detector the pulses are added in a nonlinear manner. The circuit elements used to provide the nonlinear adäition are shown in figure 10 .

The cathode followers are biased so that maximum rated current is drawn in the absence of an input signal. In the arrangemont shown, the cathode coupling resistors are relatively large but the cathode impedance of the tubes is maintained at a minimum. The negative going input pulses have large amplitude and drive the grids well beyond cutoff. When the tubes are cut off the output impedance rises to the value of the cathode coupling resistors.

The change in impedance of each tube is of the order of 10:1. Consequently, the output voltage which is supplied to the threshold detector is relatively small unless all four input pulses are in coincidence.

The triggers which mark the axis crossings are fed into three identical sets of tapped delay lines. These delay lines are shown functionally in figure 4 


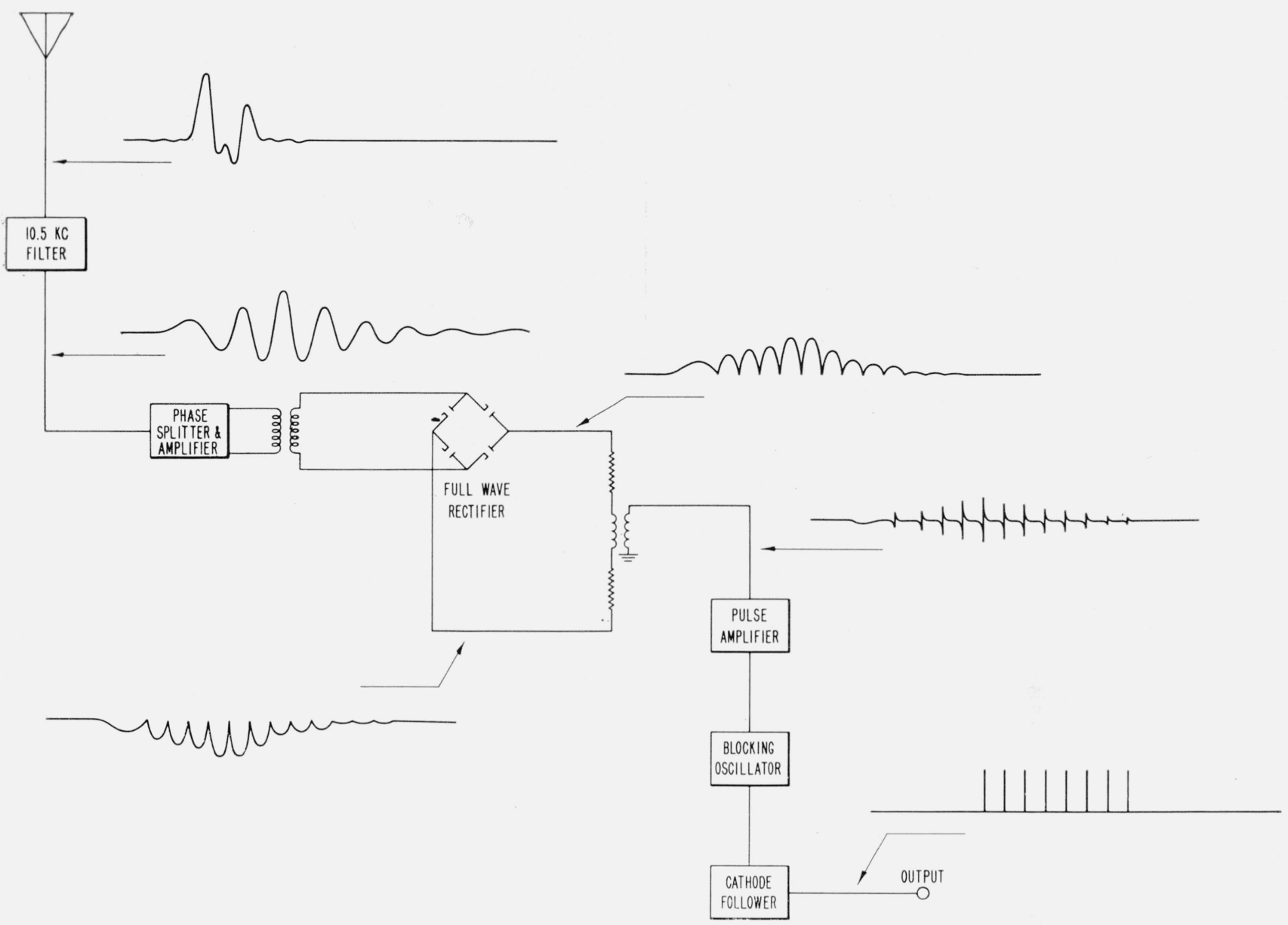

Figure 8. Axis crossing-detector.

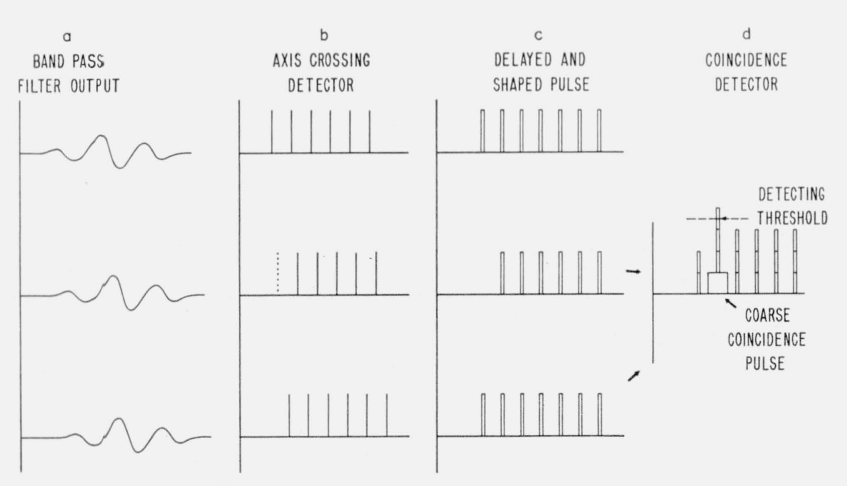

FIGURE 9. Coincidence detection.

while the actual lines used in the experimental equipment may be seen in the left hand cabinet in figure 5. The location of the taps is determined by the increment of azimuth desired between adjacent sectors and the time difference functions shown in figure 2. The desired combination of taps is selected by three switches mounted on a common shaft. An example of the delay values required for $6 \mathrm{deg}$ increments of azimuthal switching is shown in figure 11.

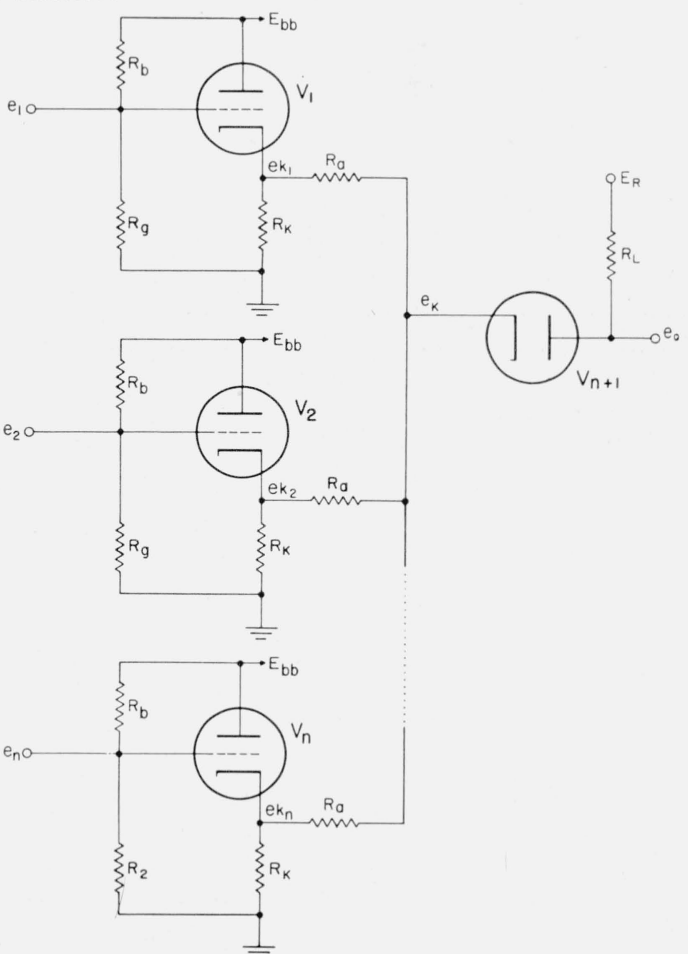

Figure 10. Variable impedance coincidence circuit. 

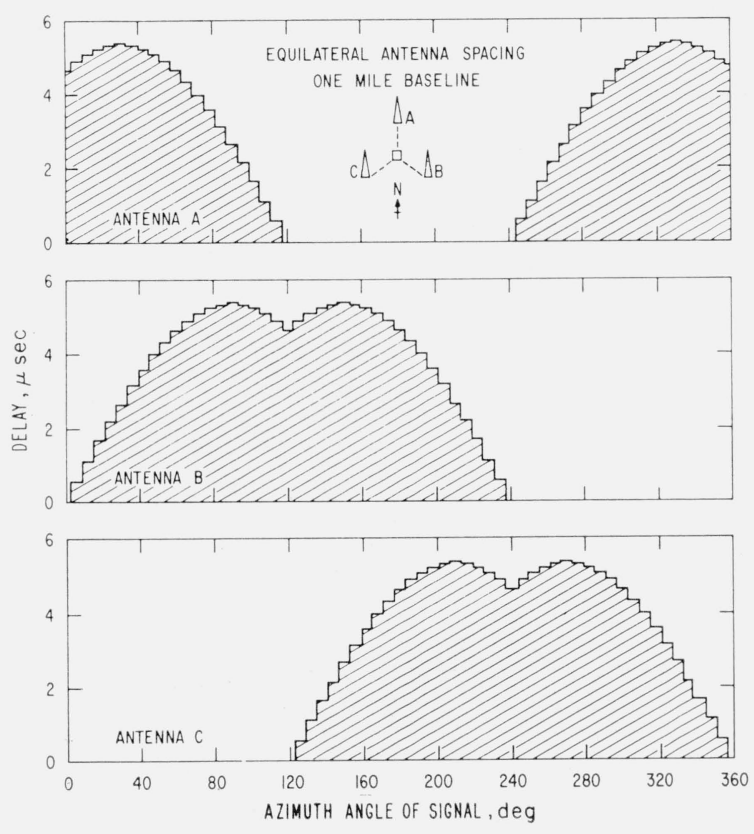

Figure 11. Example of delay values required.

强 The manner in which the delay lines are tapped is rather unique and is quite important from a standpoint of making the instrumentation practical. The delay line used is of the distributed constant, magnetic core type, which is commercially available in a wide range of delay and bandwidth characteristics. For this application, a particular virtue of the delay line is that it is not perfectly shielded. Consequently, when pulses at high amplitude are fed into the line they can be detected at any desired delay by a coil placed around the outside of the delay line. There is an attenuation of the order of $40 \mathrm{db}$ between the delay line input and the pickup coil, but this loss is easily recovered by appropriate amplification.

The outstanding advantages of this method of tapping the delay line are that the taps can be located precisely at the desired delay point simply by moving them along the line, and (within practical limits) as many pickup coils as desired may be used on the same delay line because of the very loose effective coupling between the coils and line. Figure 12 is a photograph of the delay line and pickup coils for one antenna in the experimental equipment.

The location of the delay lines relative to the control panel at the Brighton Ephi station is shown in figure 5. The azimuth control switches for three sectors are located in the rack next to the delay lines. Each is a 3 -section switch which controls the delay increments for all 3 antennas. Any number of additional sectors could be operated without additional delay lines or pickup coils, but each would require a separate amplifier, pulse shaper, coincidence detector, and another 3 -section switch. The Brighton Ephi equipment provides for switching at 6-deg intervals of azimuth, however switching could be

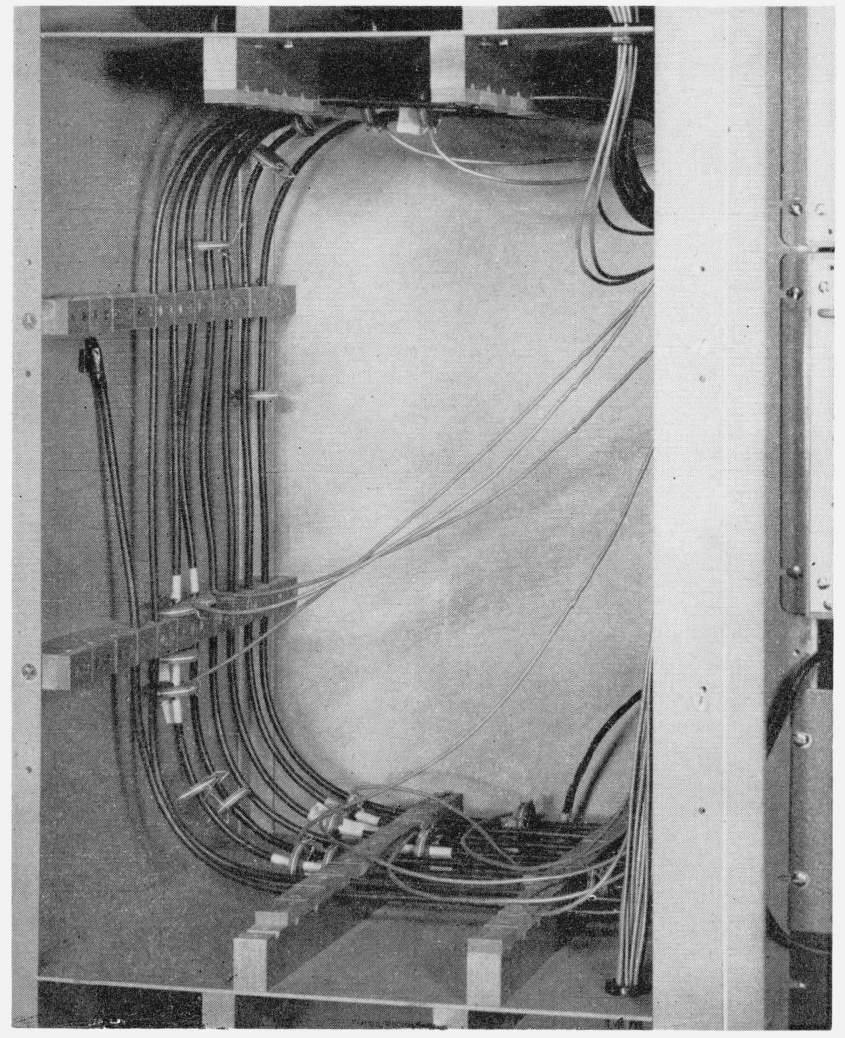

Figure 12. Pickup coils on delay line.

done at other intervals, with the proper number and placement of the pickup coils.

The pulses or triggers from any desired combination of pickup coils are amplified and used to trigger three identical, monostable multivibrators. These multivibrators produce the rectangular waveforms discussed earlier in connection with figure 3 . In the discussion of system geometry it was assumed that these waveforms were perfectly rectangular. In practical circuitry the rise and fall times are finite but sufficiently short to warrant the assumption. In the experimental equipment these times are approximately $0.1 \mu$ sec and the duration of the waveforms is adjustable from approximately 0.2 to 7.0 $\mu$ sec. With a 4-mile baseline (such as is used at Brighton) this is equivalent to a 1 to $40 \mathrm{deg}$ range in the choice of sector widths.

It is evident that all the circuitry in the three separate channels from each antenna to the input of the coincidence detector is critical with respect to phase and time delays. While corresponding circuits are made as nearly identical as possible, each channel is provided with a separate phasing adjustment so the overall transmission times can be made identical. These phasing adjustments are made as follows:

A pulse signal generator, at the centrally located equipment is permanently connected to each preamplifier (see fig. 6) through coaxial cables of identical electrical length. The signal generator pulses, therefore, arrive at each antenna simultaneously. 
This condition is equivalent to the occurrence of signals on the perpendicular bisectors of the three baselines defined by the antennas. Therefore, six headings at a mutual spacing of $60 \mathrm{deg}$ are defined at which the different pairs of pulses at the input to the coincidence detector must be coincident. The phasing adjustments are used to achieve this condition.

At the same time the phasing adjustments are made the sensitivity of the three channels is also equalized. In sferic studies it is frequently desirable to measure simultaneously rates of occurrence relative to several threshold sensitivities. In order to do this the sensitivity of the sector channels is set equal to or slightly greater than the maximum sensitivity needed while the various threshold values are established separately.

\section{Discussion}

The Ephi system of direction finding is capable of appreciably greater accuracy than systems using crossed-loop antennas. Correlation of data obtained at the Brighton station with visual and radar weather observations has shown a high degree of bearing accuracy, but until a second Ephi station is completed, or a suitable artificial sferics generator becomes available, it will be difficult to make a precise determination of bearing errors. Present indications are that these errors will amount to less than 1 degree except for signal sources at very short distances from the Ephi station.
In addition to applications of a strictly directionfinding nature (such as the location of thunderstorm areas, hurricanes, etc.) the Ephi system provides a versatile tool for more general investigations of sferics. The triggers produced in the equipment when a sferic arrives from an accurately defined sector facilitate the use of oscilloscopes, counters, and other circuitry for studies such as the following:

1. Observation and photography of sferic waveforms.

2. Directional noise studies, or sferic rates as a function of azimuth.

3. Special analyses of the amplitude and phase of sferics.

4. Correlation of sferics with particular types of meteorological phenomena.

5. Propagation studies using transient signals.

When several sectors are used additional identifying or coding information can be obtained which further extends the variety of analyses possible.

The authors acknowledge the contributions of R. H. Doherty and E. L. Berger in the equipment development and the assistance of C. A. Samson in the preparation of this paper.

Most of this work was done for the Air Force Cambridge Research Center, Air Research and Development Command, CSO\&A No. 58-28 (AFCRC). 